\title{
Detection of Kidney Abnormalities in Noisy Ultrasound Images
}

\author{
Shruthi B \\ Dept. of Computer Science and \\ Engg. \\ Sri Siddhartha Institute of \\ Technology \\ Tumkur, Karnataka
}

\author{
S Renukalatha \\ Dept. of Computer Science and \\ Engg. \\ Sri Siddhartha Institute of \\ Technology \\ Tumkur, Karnataka
}

\author{
M Siddappa \\ Dept. of Computer Science and \\ Engg. \\ Sri Siddhartha Institute of \\ Technology \\ Tumkur, Karnataka
}

\begin{abstract}
Ultrasound imaging is a widely used and safe medical diagnostic technique, due to its non-invasive nature, low cost and capability of forming real time imaging. Ultrasound imaging uses high-frequency sound to image internal structures by differing reflection signals produced when a beam of sound is projected into the body and bounces back at interfaces between the structures. However the usefulness of ultrasound imaging is degraded by the presence of signal dependant noise known as speckle. The speckle pattern depends on the structure of the image tissue and various imaging parameters. Speckle noise present in ultrasound image affects edges and fine details which limit the contrast resolution and make diagnostic more difficult. There are two main purposes for speckle reduction in medical ultrasound imaging (1) to improve the human interpretation of ultrasound images (2) despeckling is the preprocessing step for many ultrasound image processing tasks such as segmentation and registration. A number of methods have been proposed for speckle reduction in ultrasound imaging.
\end{abstract}

\section{Keywords}

Ultrasound image, Speckle noise, despeckling, kidney, filters.

\section{INTRODUCTION}

Ultrasound diagnosis differs from radiologic diagnosis in that there is no ionizing radiation involved. This is also called as ultrasound diagnosing [11]. Ultrasound can be used for medical imaging, detection, measurement and cleaning. Ultrasound imaging plays crucial roles in medical field to estimate kidney size, position, and appearance and helps to detect structural abnormalities as well as the presence of cysts, stones, cancer, congenital anomalies, swelling, blockage of urine flow etc. But presence of speckle noise and low contrast in ultrasound images, detection of kidney is a difficult as well as challenging task. Speckle is a granular noise that inherently exists and degrades the quality of the active radar, synthetic aperture radar (SAR) and medical ultrasound images.

Speckle noise present in ultrasound image affects edges and fine details which limit the contrast resolution and make diagnostic more difficult. Speckle noise is a multiplicative noise which is difficult to remove the multiplicative noise as compared to additive noise. So speckle noise is converted to additive noise by applying log transformation. Thus, speckle noise can be removed from ultrasound image. Several experiments were conducted to evaluate the proposed despeckling model. The performance metrics used are (i) Peak Signal to Noise Ratio (PSNR) and (ii) Denoising Time. PSNR is a quality measurement between the original and a denoised image. The higher the PSNR, the better is the quality of the compressed or reconstructed image. Denoising time denotes the time taken for the algorithm to perform the despeckling procedure.

Considering internal organ as kidney, kidneys are retroperitoneal organs, located near the middle of the back, just below the rib cage, one on each side of the spine. Many people are affected by chronic kidney failure due to diabetes mellitus and hypertension etc. Worldwide research indicates that one out of 10 adults has kidney problems and by 2015 it is estimated that about 36 premature deaths due to kidney disease will happen [5]. Since kidney function impairment can be life threatening, diagnosis of the disorders and diseases in the early stages is crucial. Ultrasound is one of the noninvasive low cost widely used imaging techniques for diagnosing kidney diseases.

Ultrasound image is adaptable, transferable and comparatively safe, but this type of image consists of full of acoustic interferences (speckle noise) and artifacts. Speckle is a complex phenomenon, which degrades delectability of target organ and reduces the contrast. It affects the human ability to identify normal and pathological tissue.

\section{RELATED WORK}

In [3], kidney ultrasound imaging is used to estimate kidney size and position, and help to diagnose structural abnormalities as well as the presence of cysts and stones. However, due to the presence of speckle noise in these, performing the segmentation methods for the kidney images were very challenging and therefore, deleting the complicated background will speed up and increases the accuracy of the segmentation process. Therefore, this study proposes an automatic Region of Interest (ROI) generation for kidney ultrasound images. Firstly, for speckle noise reduction the techniques such as median filter, Wiener filter and Gaussian low-pass filter are applied. Then texture analysis is performed by calculating the local entropy of the image, continued with the threshold selection, morphological operations, object windowing, determination of seed point and last but not least the ROI generation will be done. This method has performed on several kidney ultrasound images with different speckle noise reduction techniques and different threshold value selection. Based on the result, it shows that for median filter, threshold value of 0.6 has given the highest TRUE ROIs which are $70 \%$. For Wiener filter, threshold value of 0.8 has given highest TRUE ROIs which are $80 \%$ and for Gaussian low-pass filter, threshold value of 0.7 has given highest TRUE ROIs which are $100 \%$. As the result of experiment, for 
longitudinal kidney images, out of 120 images, 109 images generate true ROI (91\%) and another 11 images generate false ROI (9\%).For transverse kidney images, out of 100 images, 89 images generate true ROI (89\%) and 11 images generate false ROI(11\%).

Thus, texture analysis by using entropy filter is better compared to range filter and standard deviation filter. By choosing Gaussian low-pass filtering for speckle noise reduction technique with the threshold value of 0.7 can be applied to the rest of the images. It can be used to automatically detect the seed point of the kidney and successfully generate true kidney ROI until 89\%. The generation of true ROI is used as the preprocessing methods for any other segmentation techniques. This ROI generation can make the next image processing methods faster as some part of the original image has been cropped.

In [4], the image processing can be used to automatically detect the centroid of human kidney. The software MATLAB consists of speckle noise reduction, Gaussian filter, texture filter and morphological operators which are used for image segmentation in order to extract important features. For the result, median filter has been chosen as speckle noise reduction techniques as it is faster and detects kidney centroid better compared to wiener filter, wavelet filter and speckle noise anisotropic diffusion (SRAD) filter. This software can detect centroid up to $96.43 \%$ of accuracy. The detected centroid used in the ultrasound machine can be used as segmentation tool to reduce human errors and time. It can also be used to detect the kidney's contour automatically and time taken to process will be less.

In further research, improvement can be made by selecting another better filter, as well as by investigation the morphological operation in finding the optimum values for detecting a more accurate kidney centroid.

In [5], the variation in kidney sizes can be associated with different kidney diseases. However, the precision and accuracy of the result is low due to the manual measurement that is highly dependent on the skill and experience of the doctor. Hence an automatic measurement system is used to measure the kidney size automatically from ultrasound image. First, samples of kidney ultrasound image are collected and analyzed. Then, programming algorithm which is a combination of noise filtering method such as Gabor filter, Wiener filter, and sharpening methods are used to suppress the speckle noise on the ultrasound image while preserving the fine details. The kidney is then segmented from the image using Level set method in which the zero level set is evolved to minimize the overall energy function depending on the gradient flow. Comparison of pixel value is used to determine the maximum and minimum point which is utilized to find the length, width, and thickness. At last, volume of the kidney is calculated using ellipsoid formula.

Thus, it is possible to improve the quality of kidney ultrasound image by reducing the speckle noise and enhancing the boundary by implementation of automatic segmentation method using the level set method to partition the kidney from the ultrasound image. Testing and optimizing process are done continuously to ensure the robustness of the algorithm until the satisfied result is obtained.

In future, a better segmentation method can be used to partition the kidney without the need of a very clear image from ultrasound machine.
In [6], an algorithm is described for cleaning speckle noise in ultrasound medical images. Mathematical Morphological operations are used in this algorithm. This algorithm is based on Morphological Image Cleaning algorithm (MIC) designed by Richard Alan Peters II. The algorithm uses a different technique for reconstructing the features that are lost while removing the noise. For morphological operations it also uses arbitrary structuring elements suitable for the ultrasound images which have speckle noise.

Thus, the MMIC is the best algorithm for cleaning speckle noise which also preserves features in the image. The image cleaned by this algorithm could be used as an input for other image processing tasks such as segmentation, feature extraction, classification etc.

In [7], the kidney volume is considered as the most precise indicator of kidney size. However, it is not widely used clinically as its measurement is difficult due to the complex kidney shape. A method is used to evaluate the normal kidney volume in young Korean men by using Multi-Detector Computed Tomography (MDCT). The materials and methods are used to review MDCT data of young Korean men (113patients). After data processing, the volume and length of the kidneys are measured. Further, body parameters (height, body weight, body-surface area, and total body water) and laboratory data are collected. Glomerular Filtration rate (GFR) is calculated using Cockcroft-Gault (CG) equation. Thus, the kidney volume measured with MDCT is correlated well with body parameters, and is useful to predict renal function.

Thus, the kidney volume is a better indicator of body parameters and predictor of renal function than kidney length, suggesting that kidney volume is more useful than kidney length in clinical field. The kidney volume is more reliable index of kidney size than kidney length, and measurement of kidney volume with MDCT is useful method. Hence, MDCT facilitates measurement of kidney volume and enables to use it in clinical field.

In [8], medical image processing image denoising has become a very essential exercise all through the diagnose. In ultrasound images, the noise can restrain information which is valuable for the general practitioner. A wavelet-based thresholding method is used for noise suppression in ultrasound images. Quantitative and qualitative comparisons of the results obtained by the wavelet-based thresholding method achieved from the other speckle noise reduction techniques demonstrate its higher performance for speckle reduction.

A context-based model is introduced for adaptive threshold selection within a wavelet thresholding framework. The estimations of local weighted variance with appropriately chosen weights are used to adapt the threshold.

The proposed thresholding technique out performs all the standard speckle filters, Wiener filter Visu shrink, and Bayes shrink methods. However, by visual inspection it proves that the denoised image does not suffer with no degradation in sharpness, while removing a substantial amount of noise.

\section{METHODOLOGY}

\subsection{Model of Speckle Noise}

An inherent characteristic of ultrasound imaging is the presence of speckle noise. Speckle noise is a random and deterministic in an image [9]. Speckle has negative impact on ultrasound imaging, Radical reduction in contrast resolution may be responsible for the poor effective resolution of 
ultrasound as compared to MRI. In case of medical literatures, speckle noise is also known as texture. Generalized model of the speckle is represented as

$$
g(n, m)=f(n, m) * u(n, m)+\xi(n, m)
$$

Where, $g(n, m)$ is the observed image, $u(n, m)$ is the multiplicative component and $\xi(n, m)$ is the additive component of the speckle noise. Here $n$ and $m$ denotes the axial and lateral indices of the image samples.

For the ultrasound imaging, only multiplicative component of the noise is to be considered and additive component of the noise is to be ignored. Hence, equation (1) can be modified as

$$
\begin{aligned}
& g(n, m)=f(n, m) * u(n, m)+\xi(n, m)-\xi(n, m) \\
& \text { Therefore, } g(n, m)=f(n, m) * u(n, m)
\end{aligned}
$$

\subsection{Noise in Ultrasound Images}

Ultrasound imaging system is widely used diagnostic tool for modern medicine [9]. It is used to do the visualization of muscles, internal organs of the human body, size and structure and injuries. Obstetric sonography is used during pregnancy. In an ultrasound imaging speckle noise shows its presence while doing the visualization process.

\subsection{Filtering Techniques}

\subsubsection{Median Filter}

Median filter, one of the nonlinear filter types is created by replacing the median of the gray values of pixels into its original gray level of a pixel in a specific neighborhood. Median filter can help in reducing speckle noise as well as salt and pepper noise. The noise-reducing effect of the median filter depends on the neighborhood's spatial extent and the number of pixels involved in the median calculation.

\subsubsection{Wiener Filter}

Wiener filter inverts the blurring and removes the additive noise simultaneously by performing an optimal tradeoff between inverse filtering and noise smoothing. Besides, Wiener filtering is optimal in terms of the mean square error, where it minimizes the overall mean square error in the process of inverse filtering and noise smoothing. Wiener filtering is also a linear estimation of the original image. The Wiener filter in the frequency domain is as in equation (3)

$$
\frac{w(f 1, f 2)=H^{*}(f 1, f 2) S_{x x}(f 1, f 2)}{|H(f 1, f 2)|^{2} S_{x x}(f 1, f 2)+S_{\eta \eta}(f 1, f 2)}
$$

Where $S_{x x}(f 1, f 2), S_{\eta \eta}(f 1, f 2)$ are respectively power spectra of the original image and the additive noise, and $H(f 1, f 2)$ is the blurring filter.

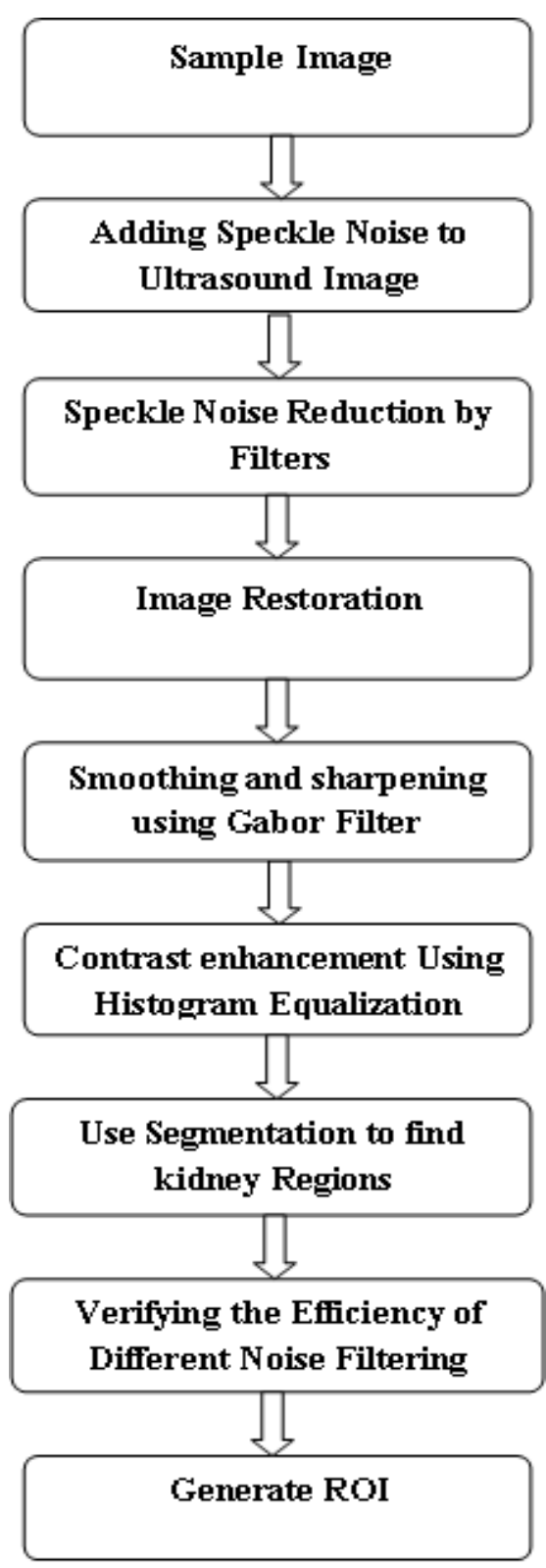

Fig 1: Block diagram of detecting Kidney abnormalities by segmentation method and ROI

\subsection{Pre-processing}

Pre-processing deals with speckle noise and low contrast. This is divided into three steps which are described according to their order of use in this system.

1. Image restoration

2. Smoothing and sharpening using Gabor filter.

3. Contrast enhancement using histogram equalization.

\subsubsection{Image Restoration}

The aim of image restoration is to remove or reduce the degradations that have occurred while the digital image was being obtained. In this system a level set function is used for proper orientation. In plain curvature motion, the curve smoothes and shrinks eventually disappear. 


\subsubsection{Smoothing and Sharpening using Gabor Filter}

Gabor filter, originally introduced by Dennis Gabor, is used to obtain the optimal resolution in both spatial and frequency domains by acting as a band-pass filter for the local spatial frequency distribution. It is widely used as convolution operator to smooth images, noise removal, and edge detection and also for segmentation of texture features especially in the fingerprint recognition. By varying the standard deviation of the Gaussian function, the degree of smoothing can be adjusted.

\subsubsection{Contrast Enhancement using Histogram Equalization}

Histogram equalization is one of the most significant parts of image analysis. It improves contrast and the goal of histogram equalization is to obtain a uniform histogram. This technique can be used on a whole image or just on a part of an image. It redistributes intensity distributions.

\subsubsection{Segmentation}

Once the original image filtered to correct the artifacts, segmentation method is used to segment the kidney region. Here two segmentation techniques are chosen, cell segmentation and region based segmentation.

Region based segmentation is simple and pixel based segmentation which uses initial seed point. Region based segmentation gives better result than cell segmentation.

\subsection{Image Quality Assessment}

To verify the efficiency of the different noise filtering methods, MSE (mean square error) and PSNR (peak signal to noise ratio) are used to evaluate the effectiveness of the noise filtering method in removing the speckle noise on ultrasound image by comparing the noise filtered image with the original image as reference. There is inverse relation between the value of MSE and PSNR where the higher the value of PSNR will result in a lower value of MSE. PSNR is normally expressed in logarithmic and the pixels are defined using eight bits per sample with value of 255 . It is actually to measure the peak error and the result is considered good if the ratio is higher which also means less noise compared to the signal (original image). The formulas used are shown as follow.

$$
M S E=\frac{1}{m n} \sum_{i=0}^{m-1} \sum_{j=0}^{n-1}[L(i, j)-K(i, j)]^{2}
$$

The PSNR (in $\mathrm{dB}$ ) is defined as

$$
\begin{aligned}
P S N R & =10 \cdot \log _{10}\left(\frac{M A X^{2}}{M S E}\right) \\
& =20 \cdot \log _{10}\left(\frac{M A X}{\sqrt{M S E}}\right)
\end{aligned}
$$

The MAX is the maximum possible value of pixel where in this case we use 255 . The size of the image is $m^{*} n$. The $I$ is the resulting image while the $\mathrm{K}$ is the reference image. In this project, the speckle noise is added to the original ultrasound image at first and the different noise filters will be used to reduce the noise. The noise filtered image will then be compared to the original image and the MSE and PSNR formulas will be used to evaluate the effectiveness of the filter in smoothing the image.

SSIM (Structured Similarity Index Method) is used for measuring the similarity between two images. The SSIM index is a full reference metric and is designed to improve on traditional methods like PSNR and MSE, which have proven to be inconsistent with human eye perception.

The difference with respect to other techniques such as MSE and PSNR is that these approaches estimate perceived errors; on the other hand, SSIM considers image degradation as perceived change in structural information. Structural information is the idea that the pixels have strong interdependencies especially when they are spatially close. These dependencies carry important information about the structure of the objects in the visual scene.

\subsection{Region of Interest (ROI)}

Region of interest is used to improve the speed and accuracy of further segmentation process. Furthermore, many existing kidney ultrasound image processing methods, been developed based on a manually selected ROI, not on the whole image. ROI cuts only the redundant background while keeping the kidney and nearby surrounding tissues untouched.

\section{RESULTS and ANALYSIS}

As per the observation from below table 1, higher the value of PSNR means more noise is removed. Least MSE value indicates that slightly biased towards over smoothed. Hence, it is concluded that Wiener filter has more PSNR value and lowest MSE value.

Table 1: The MSE, PSNR and SSIM for various preprocessing methods

\begin{tabular}{|c|c|c|c|}
\hline $\begin{array}{c}\text { Types of } \\
\text { filters }\end{array}$ & MSE & PSNR & SSIM \\
\hline Median Filter & 46.0431 & 72.5295 & 0.6846 \\
\hline Wiener Filter & 39.7071 & 74.0100 & 0.6191 \\
\hline
\end{tabular}

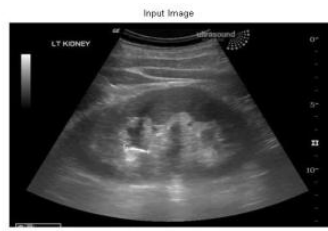

Fig 2: Input Image

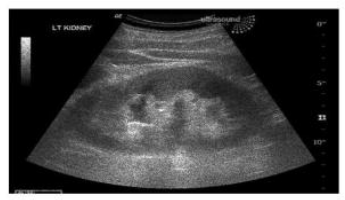

Fig 3: Speckle Noise Image 


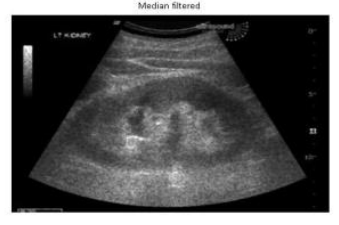

Fig 4: Median filtered image

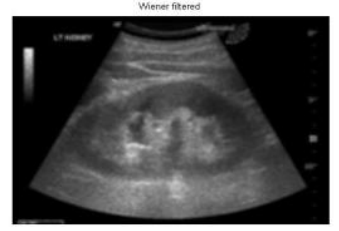

Fig 5: Wiener Filtered Image

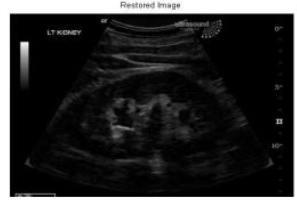

Fig 6: Restored image

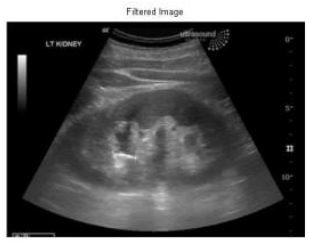

Fig 7: Gabor filtered image

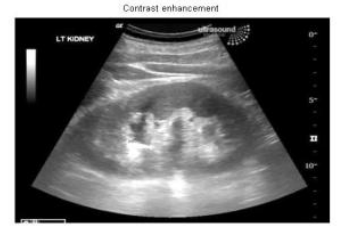

Fig 8: Contrast enhanced image

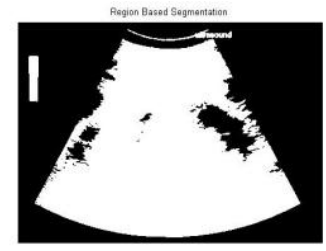

Fig 9: Region based segmentation image

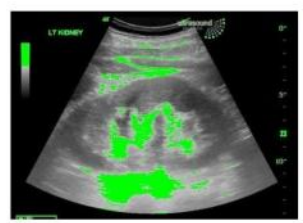

Fig 10: Cell based segmentation image

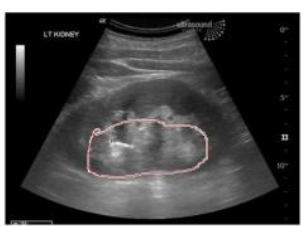

Fig 11: ROI generated by draggable freehand region

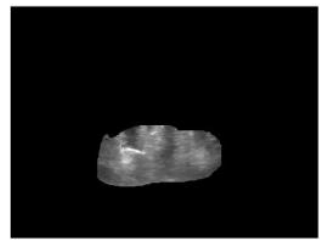

Fig 12: ROI generated image

\section{CONCLUSION}

Thus, while developing an efficient and robust denoising method for ultrasound images one has to take into account number of factors. The choice of despeckling filter and speckle model plays an important role in the design of speckle reduction methods and it differs from application to application. From the visual inspection it has seen that region based segmentation results better than cell based segmentation. A system has been developed to enhance the quality of kidney ultrasound image by reducing the speckle noise. The result shows that higher the PSNR value lower the MSE value indicating that more noise is removed. ROI (Region of Interest) is performed to detect the boundary of the region and which also improves speed and accuracy.

Further work will be done for automatic generation of ROI to find out the abnormalities and then comparing with the manually generated ROI to estimate the better result by plotting graph by using ROC (Receiver Operating Characteristic).

\section{REFERENCES}

[1] Shruthi B, S Renukalatha, Dr. M Siddappa, "Speckle noise reduction in ultrasound images - A Review", International journal of Engineering Research \& Technology, Vol. 4 Issue 02, Feb 2015.

[2] Ultrasound village [Online]. Available: $\mathrm{http}: / / \mathrm{www} \cdot$ ultrasoundvillage.com

[3] Wan M. Hafizah, Eko Supriyanto , "Automatic generation of region of interest for kidney ultrasound images using texture analysis", International Journal Of Biology And Biomedical Engineering, issue 1,Volume 6, 2012.

[4] Wan M. Hafizah, Nurul A. Tahir, Eko Supriyanto, Adeela Arooj, Syed M. Nooh, "New Technique towards Operator Independent Kidney Ultrasound Scanning", International Journal Of Computers, Issue 1,Volume 6, 2012.

[5] Eko Supriyanto, Wan Mahani Hafiza, Yeoh Jing Wui, Adeela Arooj,"Automatic Non Invasive Kidney Volume Measurement Based On Ultrasound Image", Recent Researches in Computer Science, ISBN:978-1-61804019-0. 
[6] T.Ratha Jeyalakshmi and K.Ramar," A Modified Method for Speckle Noise Removal in Ultrasound Medical Images", International Journal of Computer and Electrical Engineering, Vol. 2, No. 1, February, 2010.

[7] Shin, H.S., Chung, B. H., Lee, S.E., Kim, W.J.,Ha, H.I., Yang, C.W.," Measurement of Kidney Volume with Multi-Detector Computed Tomography Scanning in Young Korea”, Original Article, volume 50(2), 2009.

[8] S.Sudha, G.R.Suresh and R.Sukanesh, "Speckle Noise Reduction in Ultrasound Images by Wavelet Thresholding based on Weighted Variance", International Journal of Computer Theory and Engineering, Volume 1, April 2009.
[9] Milindkumar V. Sarode, Prashant R. Deshmukh, "Reduction of Speckle Noise and Image Enhancement of Images Using Filtering Technique", International Journal of Advancements in Technology, Vol 2, January 2011.

[10] Khaled Z. AbdElmoniem, Yasser M. Kadah and AbouBakr M. Youssef, "Real Time Adaptive Ultrasound Speckle Reduction and Coherence Enhancement", 078032977/00/\$10@ 2000 IEEE, pp. 172-175.

[11] Ultrasound image- Wikipedia, the free encyclopedia,http://en.wikipedia.org/wiki/ultrasound _image. 